\title{
Feature-based technique for partial fingerprint matching
}

\begin{abstract}
This paper deals with filter bank to match partial fingerprints. The method uses both local and global details in a fingerprint and defined as fixed length feature vector. Final matching is done by calculating Euclidean distance between the two corresponding feature vectors. Input fingerprints are made to $256 \times 256$ image of 8-bit grayscale with 500 dots per inch. Finger code is calculated by rotating the input images. Normalization is applied after cropping and sectoring the fingerprint image and finally gabor filters are used with same angle of rotation. Results proved that our method is better in false acceptance and total error rate when compared to the minutiae based approach. FVC2004 database is used for testing and comparison.
\end{abstract}

Keyword: Core point localization; Eucildean distance; Gabor filters 